\title{
Phytomedicine
}

Volume 20, Issues 8-9, 15 June 2013, Pages 734-742

\section{Jacaric acid and its octadecatrienoic acid geoisomers induce apoptosis selectively in cancerous human prostate cells: a mechanistic and 3-D structure-activity study}

Jihane Gasmi, J. Thomas Sanderson $\mathbf{a} \cdot \mathbf{\square}$

+ Show more

https://doi.org/10.1016/j.phymed.2013.01.012

Get rights and content

\section{Abstract}

Plant-derived non-essential fatty acids are important dietary nutrients, and some are purported to have chemopreventive properties against various cancers, including that of the prostate. In this study, we determined the ability of seven dietary C-18 fatty acids to cause cytotoxicity and induce apoptosis in various types of human prostate cancer cells. These fatty acids included jacaric and punicic acid found in jacaranda and pomegranate seed oil, respectively, three octadecatrienoic geometric isomers (alpha-and beta-calendic and catalpic acid) and two mono-unsaturated C-18 fatty acids (trans- and cis-vaccenic acid). Jacaric acid and four of its octadecatrienoic geoisomers selectively induced apoptosis in hormone-dependent ( $\mathrm{LNCaP}$ ) and -independent (PC-3) human prostate cancer cells, whilst not affecting the viability of normal human prostate epithelial cells (RWPE-1). Jacaric acid induced concentration- and time-depedent LNCaP cell death through activation of intrinsic and extrinsic apoptotic pathways resulting in cleavage of PARP-1, modulation of pro- and antiapoptotic Bcl-2 family of proteins and 